\title{
Controlling Hopf Bifurcations: Discrete-Time Systems
}

\author{
GUANRONG CHEN ${ }^{\mathrm{a}, *}, \mathrm{JIN}^{-Q I N G}$ FANG $^{\mathrm{b}}$, YIGUANG HONG $^{\mathrm{c}}$ and HUASHU QIN $^{\mathrm{c}}$ \\ a Department of Electrical and Computer Engineering, University of Houston, Houston, TX 77204, USA; \\ ${ }^{\mathrm{b}}$ China Institute of Atomic Energy, P.O. Box 275-27, Beijing 102413, P.R. China; \\ ${ }^{\mathrm{c}}$ Institute of Systems Science, Academia Sinica, Beijing 100080, P.R. China
}

(Received 20 July 1999)

\begin{abstract}
Bifurcation control has attracted increasing attention in recent years. A simple and unified state-feedback methodology is developed in this paper for Hopf bifurcation control for discrete-time systems. The control task can be either shifting an existing Hopf bifurcation or creating a new Hopf bifurcation. Some computer simulations are included to illustrate the methodology and to verify the theoretical results.
\end{abstract}

Keywords: Feedback control, Hopf bifurcation, Limit cycle, Period-doubling bifurcation, Stability

\section{INTRODUCTION}

Bifurcation control means to design a controller that can modify the bifurcative properties of a given nonlinear system, so as to obtain some desired dynamical behaviors. Typical examples include delaying the onset of an inherent bifurcation, relocating an existing bifurcation point, modifying the shape or type of a bifurcation chain, introducing a new bifurcation at a preferable parameter value, stabilizing a bifurcated periodic trajectory, changing the multiplicity, amplitude, and/or frequency of some limit cycles emerging from bifurcation, optimizing the system performance near a bifurcation point, or a certain combination of some of these $[1,2,4-6,9,10,14,17]$. Bifurcation control is important not only in its own right, but also in providing an effective strategy for chaos control. In particular, period-doubling bifurcation is a typical route to chaos in many nonlinear discrete-time dynamical systems. Bifurcation control is useful in many engineering applications, as discussed in [10].

System bifurcations can be controlled by using different methods, such as linear delayed state-feedback [7,8] or nonlinear state-feedback [2], using a washout filter [16], employing harmonic balance approximation $[4,5,11,14,15]$, and applying the quadratic invariants in the normal form [13]. In [10], a unified linear as well as simple nonlinear state-feedback technique was developed for Hopf bifurcation control for continuous-time systems. In this paper, this new methodology is further extended to discretetime systems. Discrete-time systems differ from the continuous ones in many aspects. For instance,

* Corresponding author. 
the former has the typical period-doubling bifurcation but the latter generally does not. In the investigation of this paper, both problems of shifting and creating a Hopf bifurcation are discussed. Computer simulations are included to illustrate the methodology and to verify the theoretical results.

This paper is organized as follows. Section 2 briefly summarizes the classical Hopf bifurcation theory for discrete-time systems. Sections 3 and 4 study the state-feedback control problem for Hopf bifurcations, including computer simulation results. Section 5 concludes the investigation with some discussion.

\section{HOPF BIFURCATIONS: PRELIMINARIES}

In this section, the classical criterion for discrete Hopf bifurcations is briefly reviewed.

Consider a general two-dimensional parametrized system:

$$
\begin{aligned}
& x_{k+1}=f\left(x_{k}, y_{k} ; \mu\right) \\
& y_{k+1}=g\left(x_{k}, y_{k} ; \mu\right),
\end{aligned}
$$

with a real variable parameter $\mu \in R$ and an equilibrium point $\left(x^{*}, y^{*}\right)$, satisfying $x^{*}=f\left(x^{*}, y^{*} ; \mu\right)$ and $y^{*}=g\left(x^{*}, y^{*} ; \mu\right)$ simultaneously for all $\mu$. Let $J(\mu)$ be its Jacobian at this equilibrium, with eigenvalues $\lambda_{1,2}(\mu)$ satisfying $\lambda_{2}(\mu)=\bar{\lambda}_{1}(\mu)$. If, despite some minor details,

$$
\left|\lambda_{1}\left(\mu^{*}\right)\right|=1 \quad \text { and }\left.\quad \frac{\partial\left|\lambda_{1}(\mu)\right|}{\partial \mu}\right|_{\mu=\mu^{*}}>0
$$

then the system undergoes a Hopf bifurcation at the bifurcation point $\left(x^{*}, y^{*}, \mu^{*}\right)[3,12,14]$. More precisely, in any small left-neighborhood of $\mu^{*}$ (i.e., $\left.\mu<\mu^{*}\right),\left(x^{*}, y^{*}\right)$ is a stable focus; and in any small right-neighborhood of $\mu^{*}$ (i.e., $\mu>\mu^{*}$ ), this focus changes to be unstable, usually surrounded by a limit cycle.

Here, the second condition in (2) refers to as the transversality condition for the crossing of the eigenlocus at the unit circle, namely, the eigenlocus is not tangent to the circle. Moreover, both supercritical and subcritical bifurcations can be further distinguished, via however a rather complicated series coordinate transformations (see Theorem 9.7 of [12]).

\section{CONTROLLING THE HOPF BIFURCATION}

Conceptually, Hopf bifurcations can be relatively easily created for a given two-dimensional system, in a way similar to that for the continuous-time case studied in [10]. Both calculation and simulation have confirmed this observation. Therefore, the current interest is twisted to finding out if Hopf bifurcations can also be created in a one-dimensional system in some way, which is not intuitively obvious. For this purpose, consider a parametrized one-dimensional system in a general form

$$
x_{k+1}=f\left(x_{k} ; \mu\right)
$$

with a real parameter $\mu \in R$ and an equilibrium point $x^{*}$. Clearly, this one-dimensional system cannot have a classical Hopf bifurcation due to the dimensional deficit. However, one may consider the following bifurcation control problem instead: Design a state-feedback controller, $u_{k}=u_{k}\left(x_{k} ; \mu\right)$, to be added to the right-hand side of the given system (3), such that the controlled system displays a Hopf bifurcation in the extended phase plane associated with system (3), in a sense to be further described below.

To facilitate quantitative analysis and calculation, a specific form of controller, namely, the popular delayed state-feedback controller $[7,8]$

$$
u_{k}=u_{k}\left(x_{k}-x_{k-1} ; \mu\right),
$$

is chosen in the following discussion. It is easily seen that, in principle, the methodology can be applied to other forms of controllers.

By introducing a new state variable, $y_{k}=$ $x_{k}-x_{k-1}$, the controlled system can be written 
in the following extended form:

$$
\begin{aligned}
& x_{k+1}=f\left(x_{k} ; \mu\right)+u_{k}\left(y_{k} ; \mu\right), \\
& y_{k+1}=x_{k+1}-x_{k} .
\end{aligned}
$$

If the controller (4) is designed to satisfy $u_{k}(0 ; \mu)=0$, then it will not change the original equilibrium point, $x^{*}$, of the given system (3). The controlled system has the Jacobian at $\left(x^{0}, y^{0}\right)=$ $\left(x^{*}, 0\right)$ as

$$
J(\mu)=\left[\begin{array}{cc}
f_{x} & u_{y} \\
f_{x}-1 & u_{y}
\end{array}\right]_{x=x^{*}, y=y^{0}=0},
$$

where $f_{x}=\partial f / \partial x_{k}$ and $u_{y}=\partial u_{k} / \partial y_{k}$, with eigenvalues

$$
\lambda_{1,2}(\mu)=\frac{1}{2}\left(f_{x}+u_{y}\right) \pm \frac{1}{2} \sqrt{\left(f_{x}+u_{y}\right)^{2}-4 u_{y}} .
$$

Conditions for the controller to satisfy $\lambda_{1}(\mu)=$ $\bar{\lambda}_{2}(\mu)$ and (2) are

$$
\begin{aligned}
& \left(f_{x}+u_{y}\right)^{2} \leq 4 u_{y},\left|\lambda_{1,2}\left(\mu^{*}\right)\right|=1 \text { and } \\
& \frac{\partial\left|\lambda_{1,2}\left(\mu^{*}\right)\right|}{\partial \mu}>0 .
\end{aligned}
$$

\section{SIMULATION RESULTS}

In this section, some simple but illustrative numerical examples are presented.
To show an example, the familiar Logistic map,

$$
x_{k+1}=\mu x_{k}\left(1-x_{k}\right)
$$

is used, which has equilibrium points $x^{*}=0$ and $x^{*}=(\mu-1) / \mu$. This one-dimensional system does not have Hopf bifurcation, but rather, has the wellknown period-doubling bifurcation leading to chaos as $\mu$ varies from 1.0 to 4.0 (see Fig. 1). With the controller $u_{k}\left(y_{k} ; \mu\right)=e^{\mu} y_{k}$ being added to it, the extended controlled system

$$
\begin{aligned}
& x_{k+1}=\mu x_{k}\left(1-x_{k}\right)+u_{k}\left(y_{k} ; \mu\right), \\
& y_{k+1}=x_{k+1}-x_{k},
\end{aligned}
$$

satisfies all the stated conditions, and, as expected, has a Hopf bifurcation at $\mu^{0}=0$. The corresponding simulation results are shown in Figs. 2-4, where in Fig. 2: $\mu=-0.01<0.0=\mu^{0}$, the controlled system has a stable focus, $\left(x^{*}, y^{*}\right)=(0,0)$; in Fig. 3: $\mu=0.0=\mu^{0}$, the stable focus bifurcates to a limit cycle of period six,

$$
\begin{aligned}
\left(x^{*}, y^{*}\right)=\{ & (0.5,0.5),(0.5,0.0),(0.0,-0.5), \\
& (-0.5,-0.5),(-0.5,0.0),(0.0,0.5)\}
\end{aligned}
$$

when the initial point is $\left(x_{0}, y_{0}\right)=(0.5,0.5)$; in Fig. $4: \mu=0.01>0.0=\mu_{0}$, the focus $\left(x^{*}, y^{*}\right)=(0,0)$ becomes unstable.

This bifurcation control strategy has been tested on other systems; for instance, on the following

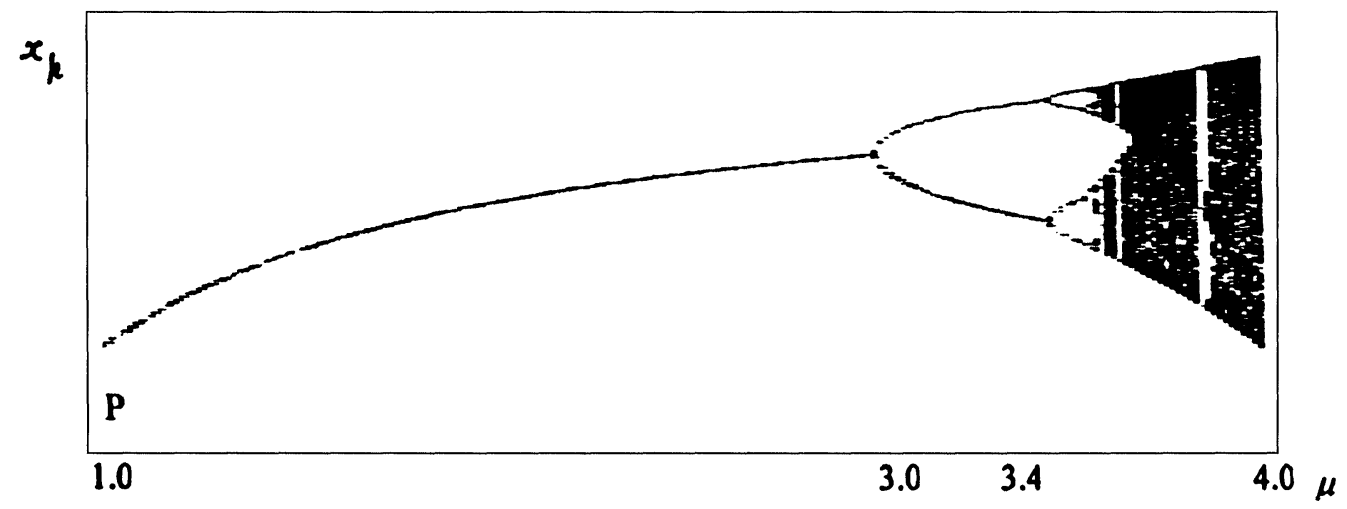

FIGURE 1 Period-doubling bifurcation of the Logistic map $(1<\mu \leq 4)$. 


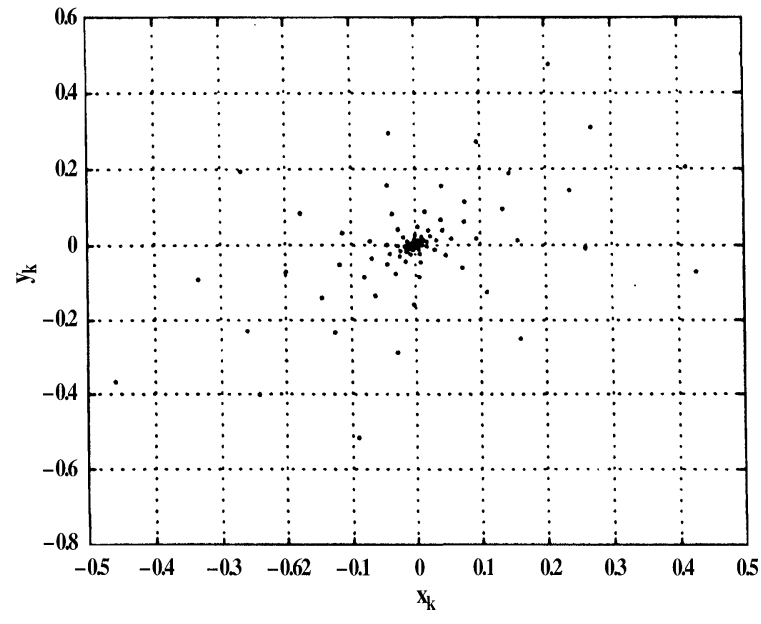

FIGURE 2 Orbit of the controlled Logistic map $(\mu=$ $\left.-0.01<0.0=\mu^{0}\right)$.

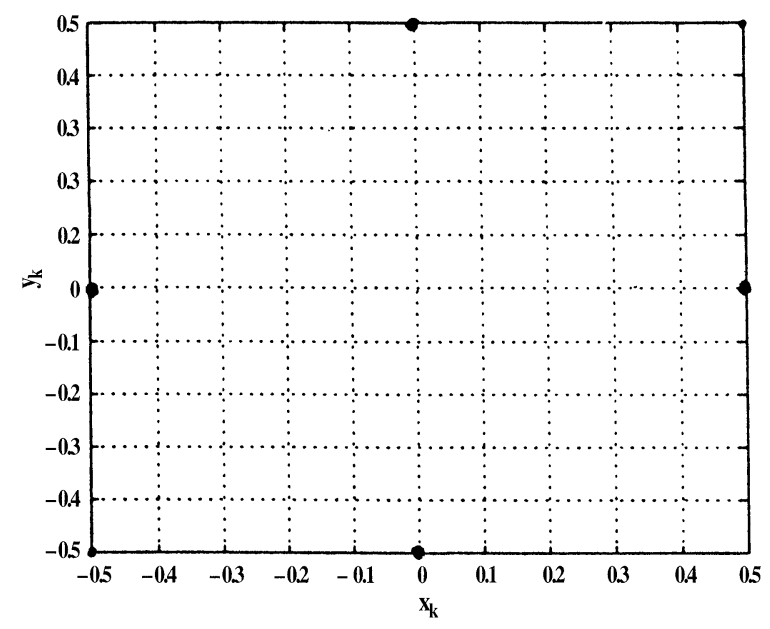

FIGURE 3 Orbit of the controlled Logistic map ( $\mu=$ $\left.0.0=\mu^{0}\right)$.

system:

$$
x_{k+1}=-\mu x_{k}-(1+\mu) x_{k}^{2} .
$$

This system also has a period-doubling bifurcations rather than a Hopf bifurcation. Yet, a new Hopf bifurcation can be created at $\left(x^{0}, y^{0}, \mu^{0}\right)=(0,0,1)$, by various feedback controllers. For example, either one of the following controllers works quite well:

$$
u_{k}=\mu y_{k}, \quad u_{k}=\mu^{2} y_{k}
$$

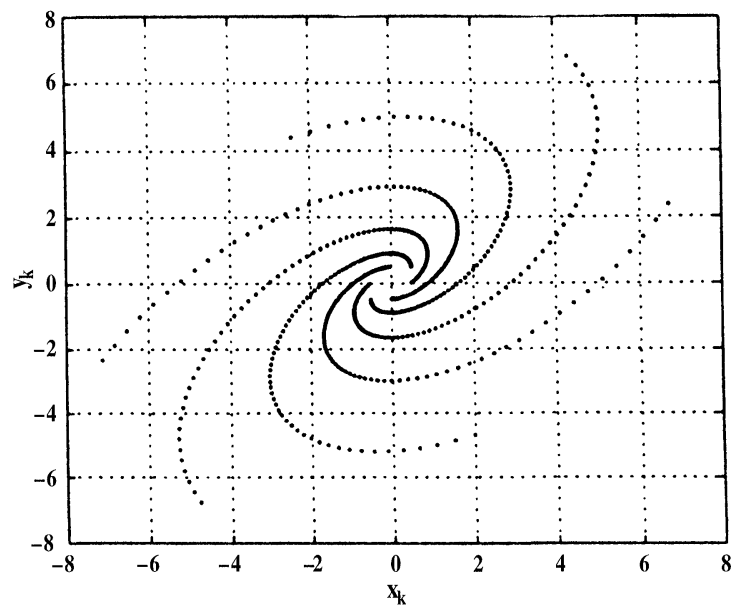

FIGURE 4 Orbit of the controlled Logistic map ( $\mu=$ $\left.0.01>0.0=\mu^{0}\right)$.

which are linear with respect to $y_{k}\left(\right.$ and $\left.x_{k}\right)$, and

$$
u_{k}=\left(2 \mu x_{k}+2 \mu+\mu^{2}\right) y_{k}
$$

which is nonlinear with respect to $\left(x_{k}, y_{k}\right)$.

\section{CONCLUSIONS}

In this paper, a simple and unified state-feedback control methodology has been developed for Hopf bifurcations for discrete-time systems, for both problems of shifting and creating a Hopf bifurcation point in the controlled system. Although the discussion here has been restricted to two-dimensional systems, the basic idea and the proposed approach can be extended to higher-dimensional dynamical systems. It is anticipated that some real applications of the new control method can be found in the near future.

\section{References}

[1] E.H. Abed and J.H. Fu, "Local feedback stabilization and bifurcation control," Sys. Contr. Lett., Part I: Hopf bifurcation, 7, 11-17, 1986; Part II: Stationary bifurcation, 8, 467-473, 1987.

[2] E.H. Abed, H.O. Wang and R.C. Chen, "Stabilization of period doubling bifurcations and implications for control of chaos," Physica D, 70, 154-164, 1994. 
[3] D.K. Arrowsmith and C.M. Place, An Introduction to Dynamical Systems, Cambridge University Press, New York, 1990.

[4] D.W. Berns, J.L. Moiola and G. Chen, "Feedback control of limit cycle amplitudes from a frequency domain approach," Automatica, 34, 1567-1573, 1998.

[5] J.L. Moiola, D.W. Berns and G. Chen, "Controlling degenerate Hopf bifurcations," Latin American Applied Research Journal, 29, 213-220, 1999.

[6] G. Chen, "Chaos, bifurcation, and their control," in The Wiley Encyclopedia of Electrical and Electronics Engineering, J. Webster (Ed.), Wiley, New York, February 1999 (in press).

[7] M.E. Brandt and G. Chen, "Bifurcation control of two nonlinear models of cardiac activity," IEEE Trans. on Circ. Sys. - I, 44, 1031-1034, 1997.

[8] G. Chen and X. Yu, "On time-delayed feedback control of chaotic dynamical systems," IEEE Trans. on Circ. Sys.-I, 46, 767-772, 1999.

[9] G. Chen and X. Dong, From Chaos to Order: Perspectives, Methodologies, and Applications, World Scientific Pub. Co., Singapore, 1998.

[10] G. Chen, J.-Q. Fang, Y. Hong and H.S. Qin, "Controlling Hopf bifurcations: Continuous-time systems," 1998 (preprint).
[11] R. Genesio, A. Tesi, H.O. Wang and E.H. Abed, "Control of period doubling bifurcations using harmonic balance," Proc. of Conf. on Decis. Contr., San Antonio, TX, 1993, pp. 492-497.

[12] P. Glendinning, Stability, Instability and Chaos, Cambridge University Press, New York, 1994.

[13] W. Kang, "Bifurcation and normal form of nonlinear control systems," Parts I and II, SIAM J. of Contr. Optim., 36, 193-232, 1998.

[14] J.L. Moiola and G. Chen, Hopf Bifurcation Analysis: A Frequency Domain Approach, World Scientific Pub. Co., Singapore, 1996.

[15] A. Tesi, E.H. Abed, R. Genesio and H.O. Wang, "Harmonic balance analysis of period-doubling bifurcations with implications for control of nonlinear dynamics," Automatica, 32, 1255-1271, 1996.

[16] H.O. Wang and E.H. Abed, "Bifurcation control of a chaotic system," Automatica, 31, 1213-1226, 1995.

[17] H. Yabuno, "Bifurcation control of parametrically excited Duffing system by a combined linear-plus-nonlinear feedback control," Nonlin. Dynam., 12, 263-274, 1997. 


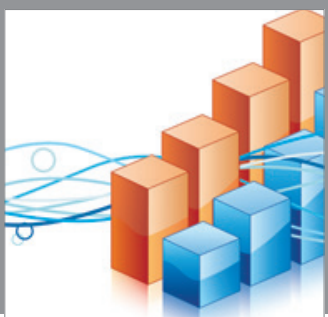

Advances in

Operations Research

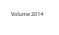

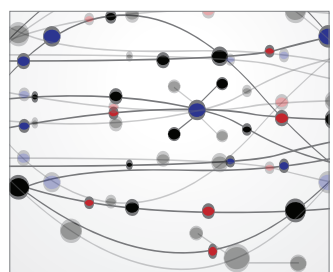

\section{The Scientific} World Journal
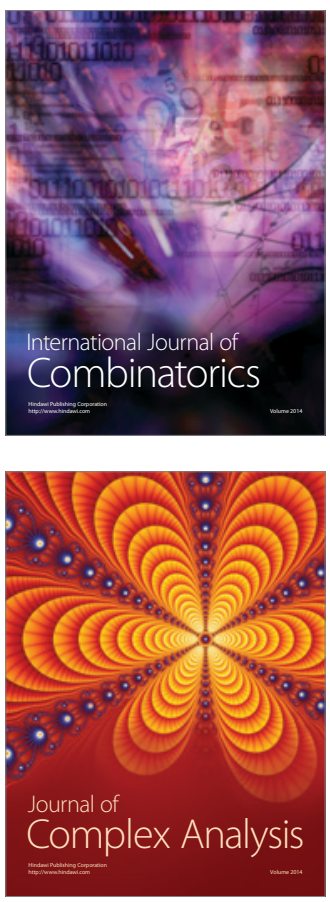

International Journal of

Mathematics and

Mathematical

Sciences
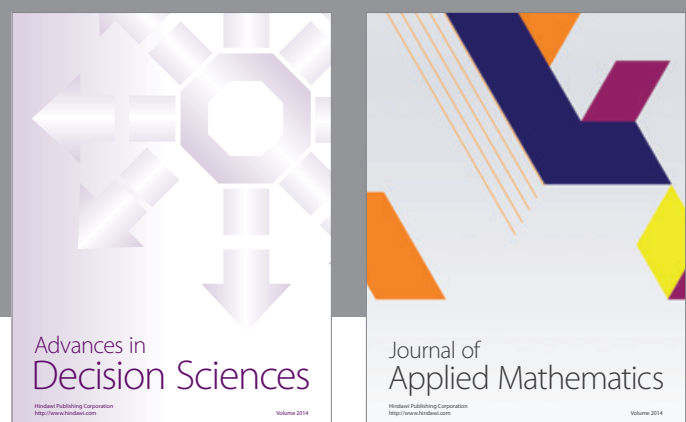

Journal of

Applied Mathematics
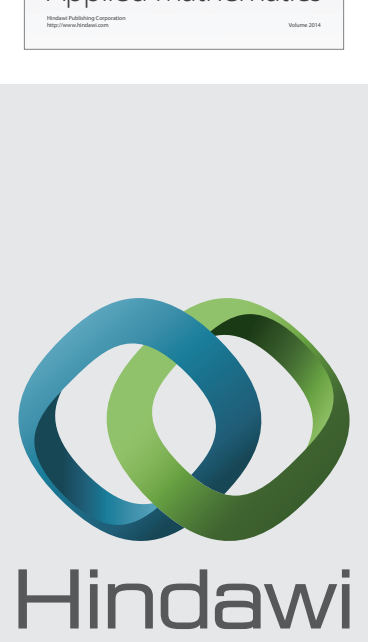

Submit your manuscripts at http://www.hindawi.com
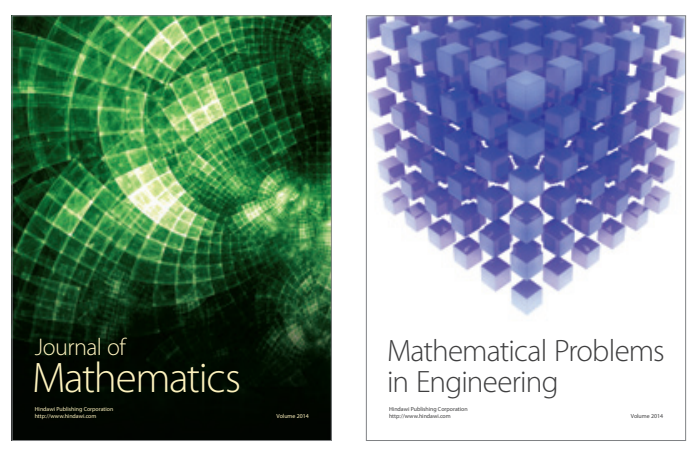

Mathematical Problems in Engineering
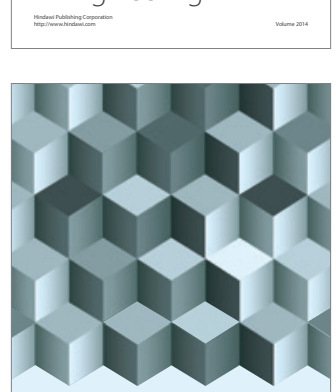

Journal of

Function Spaces
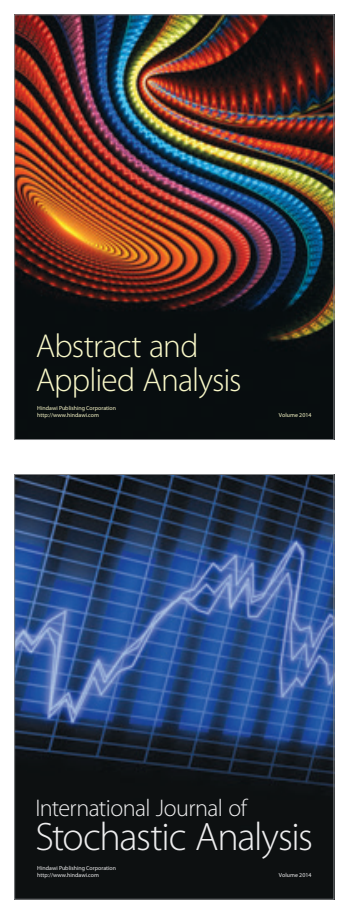

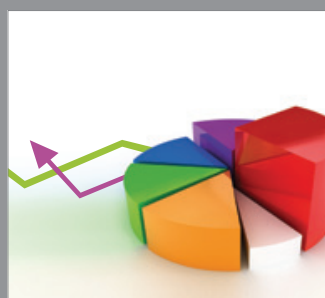

ournal of

Probability and Statistics

Promensencen
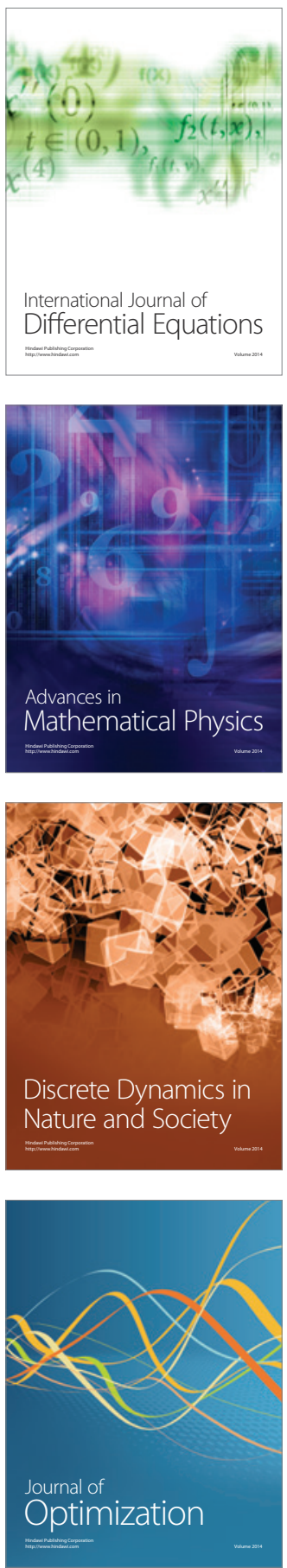